ARTÍCULOS 



\title{
ANÁLISIS SOCIODEMOGRÁFICO Y ESPACIAL DE LA MOVILIDAD LABORAL DIARÍA EN CANARIAS
}

\author{
Ma Candelaria Barrios González \\ Departamento de Economía Aplicada. Universidad de La Laguna \\ cbarrios@ull.es \\ Montserrat Hernández López \\ Departamento de Economía de las Instituciones. Estadística y Econometría.Universidad de La Laguna \\ mhdezl@ull.es
}

\section{RESUMEN}

Las mejoras en las infraestructuras de transportes han hecho posible que la movilidad diaria entre lugar de trabajo y residencia no esté condicionada, en general, por la localización de ambos en el mismo municipio. Utilizando la información del Censo de Población y Viviendas de 2001 sobre la movilidad laboral de la población ocupada canaria, se revisan sus características sociodemográficas y se realiza un análisis cluster con el objetivo de identificar grupos homogéneos de municipios canarios en cuanto a su movilidad laboral externa con la intención de explicar los motivos de un nivel alto de movilidad laboral externa en unos y no en otros.

Palabras clave: movilidad laboral, Canarias, análisis cluster.

\begin{abstract}
The improvements in the infrastructures of transport have made possible that the commuting is not determined, in general, by the location of both places in the same municipality. Making use of the information about the labor mobility of the Canary working population available in the 2001 Census of population and housings, the aim of this paper is the revision of the socio-demographic characteristics and the classification of the Canary municipalities as for his labor external mobility in order to help to explain the reasons for an external high mobility in some and not in others.
\end{abstract}

Key words: commuting, Canary Islands, cluster analysis.

Fecha de recepción: mayo 2012.

Fecha de aceptación: septiembre 2014. 


\section{INTRODUCCIÓN}

Entre los desplazamientos diarios que realiza la población, los más frecuentes y relevantes son los desplazamientos que tienen lugar entre la residencia y el trabajo. La mejora del nivel de vida, consecuencia del desarrollo económico, ha permitido que los desplazamientos diarios al trabajo se realicen cada vez a mayores distancias debido a una mayor disponibilidad de infraestructuras y medios de transporte que acortan los tiempos de desplazamiento. De esta forma, la población, si lo desea, puede mantener la inmovilidad residencial, ya que la accesibilidad al lugar de trabajo ha mejorado mucho en las últimas décadas, especialmente en relación al componente de mayor importancia del coste total del desplazamiento al trabajo, el tiempo (Godenau y Arteaga, 2003).

Las características sociodemográficas, económicas y culturales de la población determinan en gran medida su movilidad: la capacidad de desplazamiento es diferente según sea la edad, el estado civil, el género, el nivel de estudios, y la rama de actividad en la que trabaje. Además, se ha argumentado que los procesos de cambio más importantes que afectan a los espacios metropolitanos tienen que ver con sus estructuras sociodemográficas, económicas y culturales (García, 2008). Sin embargo, la diferencia clásica entre campo y ciudad no es fácil de defender hoy en día, ya que las típicas variables socioeconómicas que definían la ciudad han dejado paso a los flujos cotidianos individuales como elementos más apropiados para su definición. De esta forma, se habla de territorios funcionales en donde la identificación de orígenes y destinos por motivos de trabajo en los censos de población tiene una especial relevancia (Gutiérrez y García, 2005).

El estudio de la movilidad laboral diaria (commuting) puede tener muchos enfoques; en este trabajo nos centremos en estudiar las características de la movilidad laboral diaria externa en Canarias. De esta forma, el concepto de movilidad laboral diaria puede ser divido en dos: movilidad laboral interna (MI) o población ocupada que vive y trabaja en el mismo municipio, y movilidad laboral externa (ME) o población ocupada que trabaja en un municipio distinto al que reside. A partir de estas definiciones, el objetivo del artículo es doble. Por un lado, conocer los rasgos socioeconómicos esenciales de la movilidad laboral en Canarias, y, por otro, establecer una agrupación de los municipios canarios en cuanto a la movilidad laboral externa.

Diferentes estudios (Albertos et al., 2007; Roquer, 2007; Salom y Casado, 2007; García, 2008) ya han constatado que las características sociodemográficas de los individuos influyen en el comportamiento desigual de la población en materia de movilidad, tanto en estudios nacionales como regionales. Como se ha comentado, será objetivo de este artículo determinar si esas desigualdades de comportamiento en la población activa respecto a determinadas características sociodemográficas también operan en Canarias, y conocer si existen diferencias relevantes con el resto del territorio nacional. Evidentemente, el hecho de que el territorio canario sea discontinuo, compuesto por 7 islas, y que en sólo dos de ellas se ubiquen las dos capitales de provincia, pudiera explicar, en principio, la aparición de aspectos diferenciadores respecto a las conclusiones de artículos referidos a todo el territorio nacional o circunscritos a alguna comunidad autónoma peninsular. Sin embargo, sólo el análisis cuantitativo de la información disponible podrá confirmar estas conjeturas iniciales.

El resto del artículo se organiza como se indica a continuación. En la siguiente sección se contextualiza el marco de análisis del mismo y la metodología empleada. Posterior- 
mente, se realiza un análisis descriptivo de los niveles de movilidad externa, según las distintas modalidades que establece cada característica sociodemográfica considerada para el caso de estudio de la Comunidad Autónoma de Canarias por islas. En la sección cuarta se describe el análisis cluster aplicado con el objetivo de establecer grupos homogéneos de municipios canarios desde la perspectiva de la movilidad laboral externa, que ayuden a identificar los rasgos esenciales relacionados con un determinado nivel de movilidad laboral externa. Y, finalmente, se expondrán brevemente las conclusiones del trabajo en la última sección.

\section{LA MOVILIDAD LABORAL COMO OBJETO DE ESTUDIO}

El estudio de la movilidad laboral diaria desde un punto de vista individual requiere de datos que en el caso de Canarias, al no existir encuestas de movilidad para la Comunidad Autónoma, no han estado disponibles hasta el Censo de Población y Viviendas de 2001 -en adelante, Censo de 2001-, donde han sido introducidas una serie de preguntas relativas a la movilidad entre el lugar de residencia y el lugar de trabajo (Roquer y Blay, 2002). Una de estas preguntas hace referencia al lugar de trabajo, de modo que se puede conocer, para la población ocupada, cuántas personas trabajan dentro del propio municipio de residencia (MI) y cuántas fuera de él (ME).

En el último Censo, el de 2011, no existe la posibilidad de obtener la información desagregada a nivel municipal, por lo que nos ha sido imposible actualizar los datos con el nuevo Censo. La metodología aplicada en el nuevo Censo, que obtiene la información censal a partir de una gran encuesta por muestreo para conocer las características de las personas y viviendas, impide por secreto estadístico que podamos obtener información tan desagregada como ocurre con el Censo de 2001.

Con los datos procedentes de las preguntas relativas a la movilidad diaria al trabajo pretendemos estudiar las características sociodemográficas de la movilidad residencia-trabajo en Canarias por islas, distinguiendo entre la provincia de Las Palmas (LP), compuesta por las 3 islas más orientales -Gran Canaria, Lanzarote y Fuerteventura-, y la de Santa Cruz de Tenerife (SCTF), formada por las 4 restantes islas occidentales -Tenerife, Gomera, La Palma y El Hierro. Concretamente, se pretende averiguar si existen diferencias significativas entre la movilidad interna y externa en función de algunas características sociodemográficas de la población -género, edad, nivel de estudios y rama de actividad-respecto a los datos nacionales, y establecer grupos de municipios homogéneos en lo que a su movilidad laboral externa se refiere para identificar las características principales de los municipios canarios que poseen un determinado nivel de movilidad laboral externa.

La obtención de los datos necesarios para afrontar este estudio precisa del cruce de información sobre movilidad laboral externa e interna con las referidas características sociodemográficas para los 87 municipios canarios (actualmente 88, tras la creación en 2007 del municipio de El Pinar en El Hierro), opción disponible para el usuario en la página web del Instituto Nacional de Estadística; esta información sólo es posible obtenerla con el Censo de 2001, como ya hemos comentado.

El Censo de 2001 contempló 4 preguntas sobre la movilidad entre el lugar de residencia y el de trabajo, la primera, relativa al lugar de trabajo, contemplaba cinco respuestas: el propio 
domicilio, en varios municipios, en el propio municipio, en otro municipio (habiéndose de concretar en qué municipio y en qué provincia) y en otro país (concretando también éste). Las respuestas a esta primera pregunta las agruparemos en dos, la primera y la tercera serán consideradas MI, mientras las restantes serán consideradas ME, y son las que cruzaremos con las características sociodemográficas de la población ocupada en los 87 municipios. De esta forma, en la primera parte de este trabajo trataremos de conocer si existen diferencias significativas entre la población que realiza movilidad externa en función de la variables sociodemográficas elegidas y la isla de residencia.

En España no se dispone de información sobre la movilidad laboral diaria hasta el Censo de Población de 2001. Sólo Andalucía, Cataluña, País Vasco y Valencia habían incluido dentro de los censos de población anteriores algunas preguntas sobre movilidad (Pazos, 2005). Por esta razón, no se podía disponer de información para estudiar la movilidad de la población, particularmente la movilidad por motivo de trabajo, que es uno de los elementos más importantes en la definición de la organización territorial. A partir de la incorporación de esta nueva fuente, el investigador puede acceder por primera vez al conocimiento sobre la movilidad residencia-trabajo de la población a escala municipal en cualquier comunidad autónoma (Alonso et al., 2007). Actualmente, este tipo de estudios quedarán muy limitados con las características que presenta el nuevo Censo de población, que recientemente ha publicado los resultados detallados.

Una vez obtenida la información en términos absolutos, la relevancia de las características sociodemográficas en la movilidad laboral interna y externa se ha determinado calculando el porcentaje de ME para cada una de las modalidades sobre el total de la modalidad considerada. Es decir, y a modo de ejemplo, el valor de ME para el caso de las trabajadoras canarias se ha calculado dividiendo el número total de trabajadoras canarias cuyo trabajo está fuera de su municipio de residencia entre el número total de trabajadoras canarias; del mismo modo se ha hecho cuando las ME se calculan para el ámbito municipal.

\section{ANÁLISIS SOCIODEMOGRÁFICO DE LA MOVILIDAD LABORAL EN CANARIAS POR ISLAS}

En términos generales, la ME total en Canarias con información de 2001 era de 262.736 -Tabla 1- , es decir el 38\% de los ocupados trabajaban fuera de su municipio de residencia, mientras la MI alcanzaba los 427.720 ocupados, lo que suponía el 62\% restante. Estos niveles porcentuales son muy similares a los obtenidos por Roquer (2007) para el conjunto del territorio nacional.

Tabla 1

MOVILIDAD EXTERNA E INTERNA EN CANARIAS POR PROVINCIAS

\begin{tabular}{|c|c|c|c|c|}
\hline Lugar & Población ocupada & ME (\%) & MI (\%) & ME/MI \\
\hline CANARIAS & 690.456 & $262.736(38)$ & $427.720(62)$ & 0,61 \\
\hline LPGC & 367.366 & $128.159(35)$ & $239.207(65)$ & 0,54 \\
\hline SCTF & 323.090 & $134.577(42)$ & $188.513(58)$ & 0,72 \\
\hline
\end{tabular}

Fuente: Elaboración propia. Información municipal obtenida del Censo de Población 2001. 
Por islas la mayor movilidad externa la tienen las islas mayores, Tenerife y Gran Canaria, y Lanzarote y La Palma, como se puede observar en la figura 1. No obstante, Gran Canaria, a pesar de ser una de las islas mayores, con mayor población y con el mayor número de ocupados (288.280), no es la que mayor movilidad externa presenta. En el extremo opuesto, se encuentran El Hierro, La Gomera y Fuerteventura. Estos resultados concretos, agrupados sin distinciones sociodemográficas, ya fueron expuestos en un análisis monográfico (Godenau, 2005) publicado por el CES de Canarias, en donde se estudiaron los mercados locales de trabajo en Canarias y sus condiciones de movilidad y accesibilidad dentro y entre éstos.

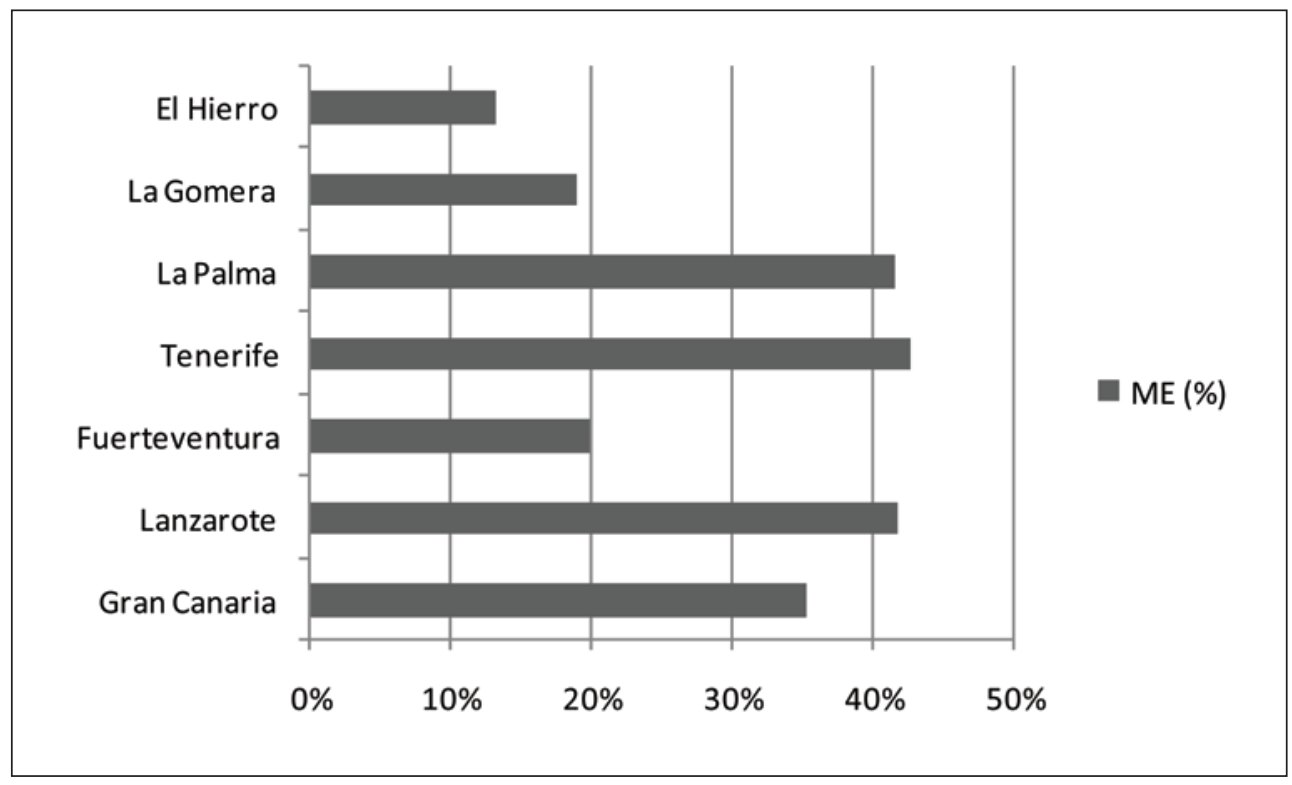

Fuente: Elaboración propia. Información municipal obtenida del Censo de Población 2001.

\section{Movilidad laboral por género: Las trabajadoras canarias muestran menor movilidad laboral externa}

El comportamiento desigual entre hombres y mujeres en cuanto a la movilidad (Casado, 2000; Salom y Delios, 1998) es un hecho conocido debido a que, aunque se han producido importantes cambios sociales que han afectado a la distribución de responsabilidades y de roles en las familias, sigue siendo mayor el porcentaje de mujeres cuyo rol familiar se circunscribe exclusivamente a las tareas domésticas, lo que limita su movilidad laboral. 


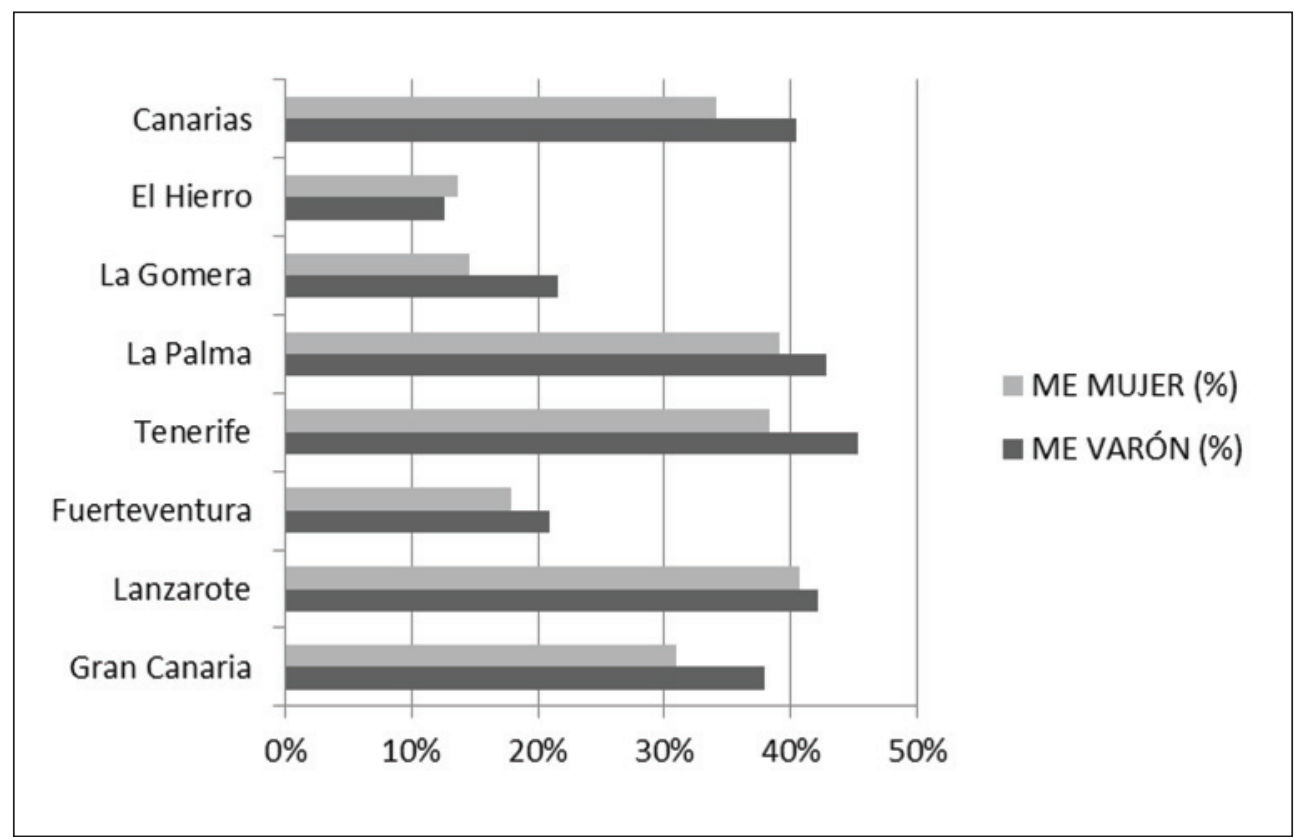

Fuente: Elaboración propia. Información municipal obtenida del Censo de Población 2001.

De hecho, las cifras de la ME masculina en Canarias son superiores a las femeninas (40\% frente a 34\%) indicando esto que de los hombres que trabajan lo hacen en mayor medida fuera de su municipio de residencia que las mujeres, circunstancia que se repite en todas las islas a excepción de El Hierro. De nuevo, se ratifica lo encontrado por otros autores respecto a este hecho para el territorio nacional (Roquer, 2007).

Los porcentajes ME tanto masculina como femenina son más elevados en Gran Canaria, Lanzarote, La Palma y Tenerife, encontrándose en el extremo opuesto El Hierro, La Gomera y Fuerteventura, con unas movilidades externas masculina y femenina que no superan el $22 \%$ y el $18 \%$, respectivamente.

\section{Movilidad laboral por edad: mayor movilidad externa entre los trabajadores canarios jóvenes}

La edad de la población es un claro condicionante en la movilidad en tanto en cuanto se ha observado que, en términos generales, existe una relación inversa entre movilidad laboral externa y edad del trabajador: la población joven está más dispuesta a desplazarse fuera de su municipio de residencia para mejorar sus condiciones laborales. Para calcular la movilidad laboral externa e interna en Canarias por grupos de edad hemos agregado las agrupaciones que aparecen en el Censo 2001 en tres grupos de edad, de 15 a 29, de 30 a 49 y mayores de 50 años, como podemos observar en la figura 3. 


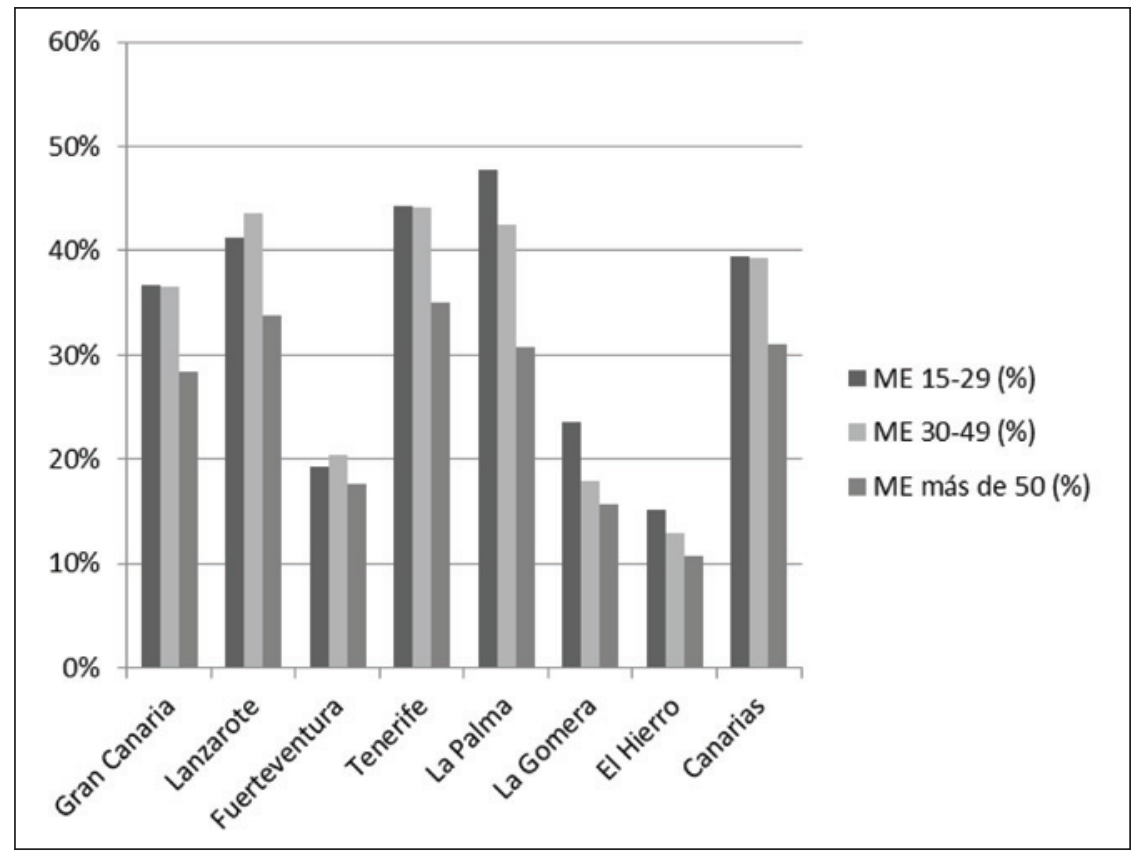

Fuente: Elaboración propia. Información municipal obtenida del Censo de Población 2001.

En lo que se refiere a la edad del trabajador canario -Figura 3-, se observa que la movilidad laboral externa en Canarias es muy similar entre los grupos de 15 a 29 años y de 30 a 49 años, descendiendo a partir de esta edad. El 80\% de los desplazamientos que por motivo trabajo se realizan fuera del municipio en Canarias se deben a los trabajadores que tienen una edad inferior a 50 años.

Si hacemos este análisis por islas, volvemos a observar un patrón de comportamiento muy similar al que obtuvimos con la variable sexo: los porcentajes ME por grupos de edad son más elevados en Gran Canaria, Lanzarote, La Palma y Tenerife, encontrándose en el extremo opuesto El Hierro, La Gomera y Fuerteventura, con una movilidad externa que no supera el 20\%, a excepción de La Gomera para el grupo de edad entre 15 y 29 años $(24 \%)$.

Comparando los resultados ofrecidos por Roquer (2007) para el conjunto nacional con los resultados obtenidos para el conjunto de la Comunidad Autónoma de Canarias, no existen diferencias relevantes, aunque sí existen cuando lo hacemos por islas.

\section{Movilidad laboral por nivel de formación: a mayor formación, mayor movilidad laboral externa}

El nivel de formación del trabajador también guarda relación directa con la movilidad laboral externa: la mayor formación suele conllevar la percepción de mayor renta, y unos 
mayores niveles de ésta permiten el acceso a mejores medios de locomoción que ayudan, finalmente, a la posibilidad de búsqueda de trabajo fuera del municipio de residencia (Miralles-Guasch y Cebollada, 2009).

En el caso canario, se han planteado 4 modalidades en la formación: «analfabetos y sin estudios» «primer grado», «segundo grado» $\mathrm{y}$ «tercer grado». La modalidad «primer grado» incluye a las personas que tienen realizada la educación básica o primaria; la de «segundo grado» agrupa a los individuos que han finalizado la educación secundaria, la ESO actual o la antigua EGB y los que tienen bachillerato o formación profesional; y la modalidad «tercer grado» está compuesta por aquellos ocupados que han realizado y acabado estudios universitarios (estudios de diplomatura, licenciatura, de doctorado o máster).

Figura 4

MOVILIDAD LABORAL CANARIA POR NIVEL DE FORMACIÓN

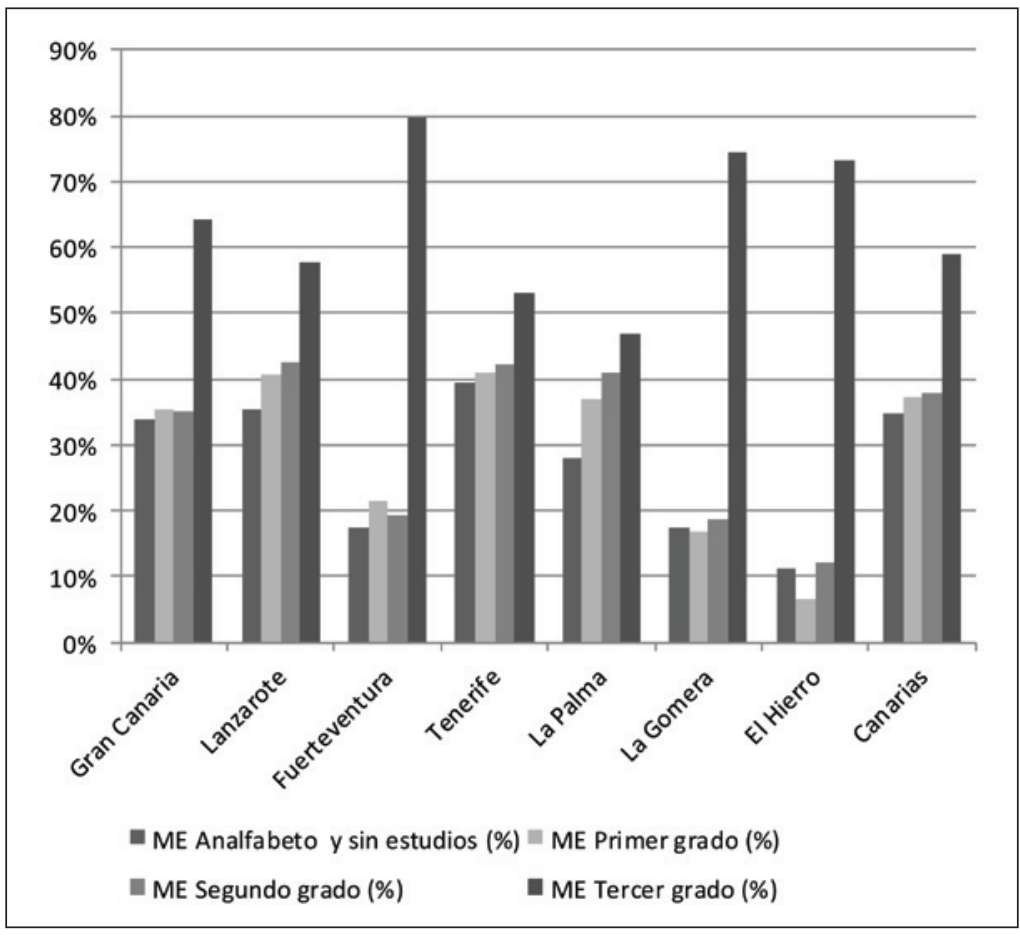

Fuente: Elaboración propia. Información municipal obtenida del Censo de Población 2001.

La mayor ME corresponde a los ocupados con mayor grado de formación, es decir, los que hemos incluido en el grupo denominado Tercer Grado, y este comportamiento es independiente de la isla que observemos: en todas es, con diferencia, el más elevado, haciéndose especialmente elevado en los casos de El Hierro, La Gomera y Fuerteventura, con una movilidad externa que supera el $70 \%$. De nuevo, estos datos confirman los resultados de otras investigaciones desarrolladas para el conjunto nacional (Roquer, 2007). 


\section{Movilidad laboral y rama de actividad: la industria canaria, sector de mayor movilidad labo- ral externa}

Las ramas de actividad elegidas para estudiar la movilidad laboral han sido el sector primario -agricultura, ganadería y pesca-, industria, construcción y servicios. En la Figura 5 se muestran los porcentajes resultantes de ME por rama de actividad. Se observa el hecho de que en Canarias la movilidad externa es claramente superior en el sector de la industria, aunque sólo representa el 7\% de la población ocupada. La razón de que esto suceda estriba en que en este sector las empresas tienen una actividad localizada en torno a polígonos industriales situados en determinados municipios.

Figura 5

MOVILIDAD LABORAL CANARIA POR RAMA DE ACTIVIDAD

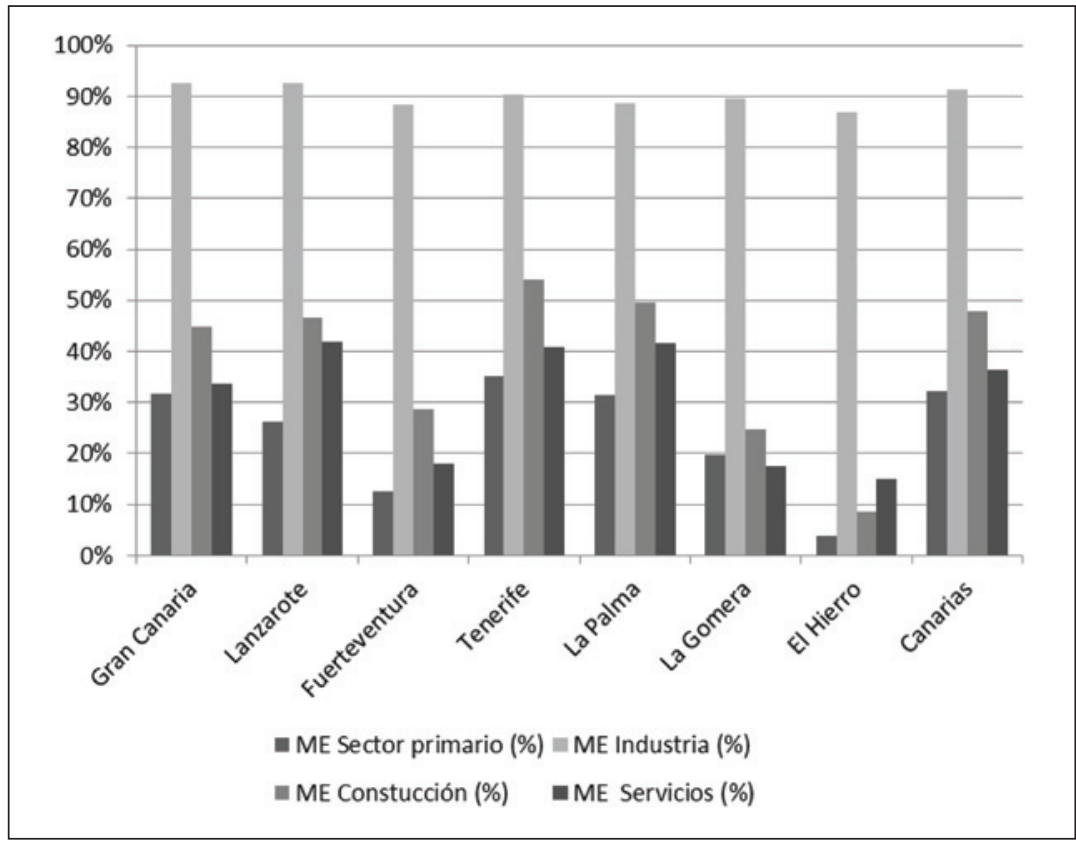

Fuente: Elaboración propia. Información municipal obtenida del Censo de Población 2001.

En el extremo opuesto, con una escasa movilidad externa se encuentra el sector primario. La baja movilidad externa en este sector se debe a que este tipo de actividad es eminentemente rural y propicia que los trabajadores ocupados vivan fundamentalmente en el municipio donde desempeñan su actividad y tienen cerca los medios de producción. La construcción es un sector que mantiene el equilibrio entre movilidad externa e interna, no ocurriendo lo mismo a nivel nacional donde la movilidad externa es claramente superior debido fundamentalmente a la concentración de la construcción en determinados lugares. Por último, el sector servicios en su conjunto mantiene una movilidad interna superior a la externa. 
Debido a la importancia que el sector servicios tiene en la Comunidad Autónoma Canaria, el 74\% de la población ocupada trabaja en este sector, y al hecho de que este dato agregado puede ocultar información relevante respecto a las actividades que incluye, se ha procedido a un segundo análisis desagregando el sector servicios en varios subsectores (en este caso para el conjunto de la Comunidad Autónoma Canaria): comercio, hostelería, transporte y comunicaciones, actividades financieras e inmobiliarias, Administración Pública, educación, sanidad y otras actividades terciarias (Figura 6).

En la figura 6 se observa que las actividades del sector servicios que presentan una mayor movilidad externa son hostelería, transporte y comunicaciones, educación y actividades sanitarias. El comportamiento de la movilidad del sector hostelería difiere del observado en el conjunto del país, donde la ME es menor (Roquer, 2007). La razón podría encontrarse en la especialización que la economía canaria tiene en el sector turístico y, como consecuencia, el mayor porcentaje de población canaria ocupada en el sector de la hostelería. A esto se añade la concentración geográfica de las actividades turísticas en determinadas zonas, donde los precios de viviendas son más elevados, obligando a la población ocupada en el sector a buscar un lugar de residencia en municipios limítrofes a los turísticos, donde el precio de la vivienda es más asequible.

Figura 6

MOVILIDAD LABORAL CANARIA POR RAMA DE SERVICIOS

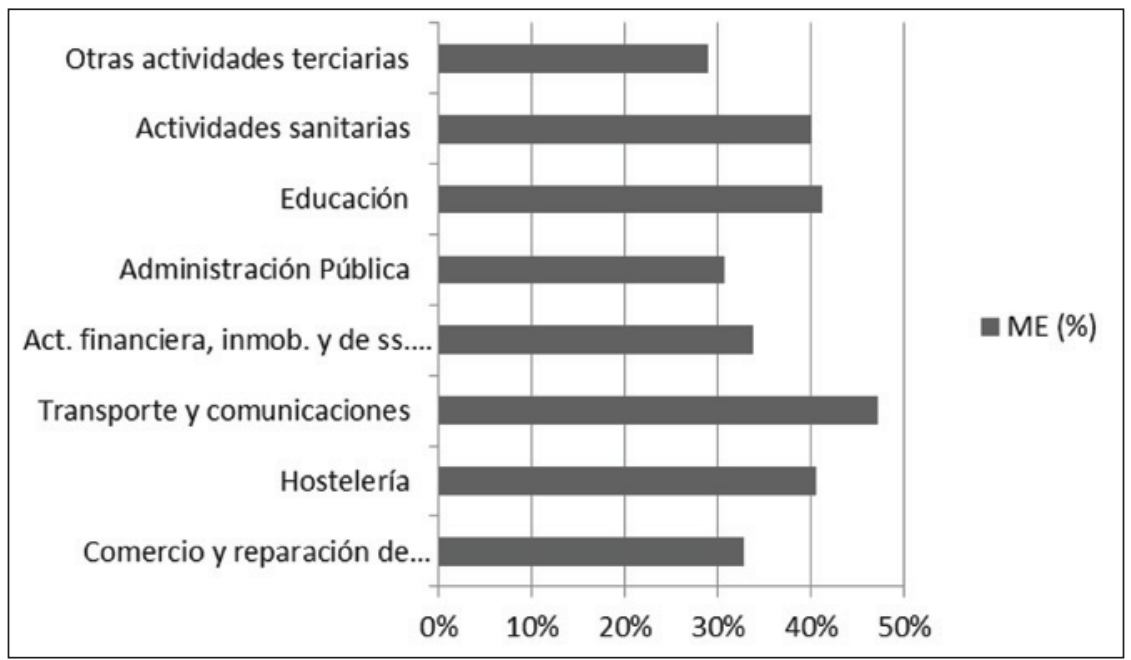

Fuente. Elaboración propia. Información municipal obtenida del Censo de Población 2001.

\section{AGRUPACIÓN DE LOS MUNICIPIOS CANARIOS RESPECTO A LA MOVILIDAD LABORAL EXTERNA}

Un primer examen de los datos porcentuales de las características sociodemográficas relacionadas con la movilidad laboral externa consideradas en este artículo hace sospechar de la posible existencia de municipios canarios más o menos parecidos en cuanto a esta 
movilidad se refiere. Sin embargo, para demostrar la existencia de estos posibles grupos de municipios se hace necesaria la aplicación de un análisis estadístico multivariante que, objetivamente, ayude a establecerlos. Las variables elegidas para este análisis son los valores porcentuales de movilidad laboral externa por municipio referidos a cada una de las modalidades de las características sociodemográficas que han sido analizadas a lo largo del artículo, además del porcentaje global de ME municipal, lo que establece una matriz final de información de 21 variables y 87 municipios.

El análisis cluster tiene por finalidad la agrupación de objetos -municipios canarios en este caso- homogéneos entre sí en cuanto a cada una de las variables que los definen (Aldenderfer y Blashfield, 1984). Dentro de los métodos cluster, existen varios métodos para la obtención de los grupos homogéneos de casos. El método usado en este caso ha sido el jerárquico aglomerativo de Ward que, a partir de la definición de la matriz de distancias euclídeas entre los objetos a clasificar respecto a los valores que tome cada uno en las características sociodemográficas que los definen, impone un orden en las fusiones entre los objetos en función del grado de similitud entre los mismos. Así, las fusiones que se van generando entre los grupos son aquellas que minimizan la suma de cuadrados de las desviaciones entre el valor de cada una de las variables que describen al objeto que se pretende fusionar en el grupo y las medias de las variables en el grupo.

En el presente caso de estudio, se ha elegido como resultado de la aplicación del método de Ward el nivel de distancia que generaba 3 grupos de municipios. El motivo de elegir este nivel de agrupación se debe a que la agrupación pretendida de municipios se basa, de hecho, en una sola variable -movilidad laboral externa del municipio-, enfocada desde distintas características sociodemográficas del municipio, de lo que parece razonable una clasificación de municipios con baja, media o alta movilidad laboral externa. Así, el estudio cuantitativo de los resultados presentados en la Tabla 2 permite constatar que cada uno se diferencia de los demás por tener un nivel porcentual diferenciado de la movilidad laboral externa. Concretamente, y en términos medios, el grupo 1 sería aquél en el que la movilidad laboral externa es porcentualmente menor; el grupo 2, el formado por municipios cuya movilidad laboral externa es media; y el grupo 3, el que aglutina a los 37 municipios de mayor movilidad externa (Figura 7).

Figura 7

MUNICIPIOS CANARIOS POR GRUPOS DE MOVILIDAD

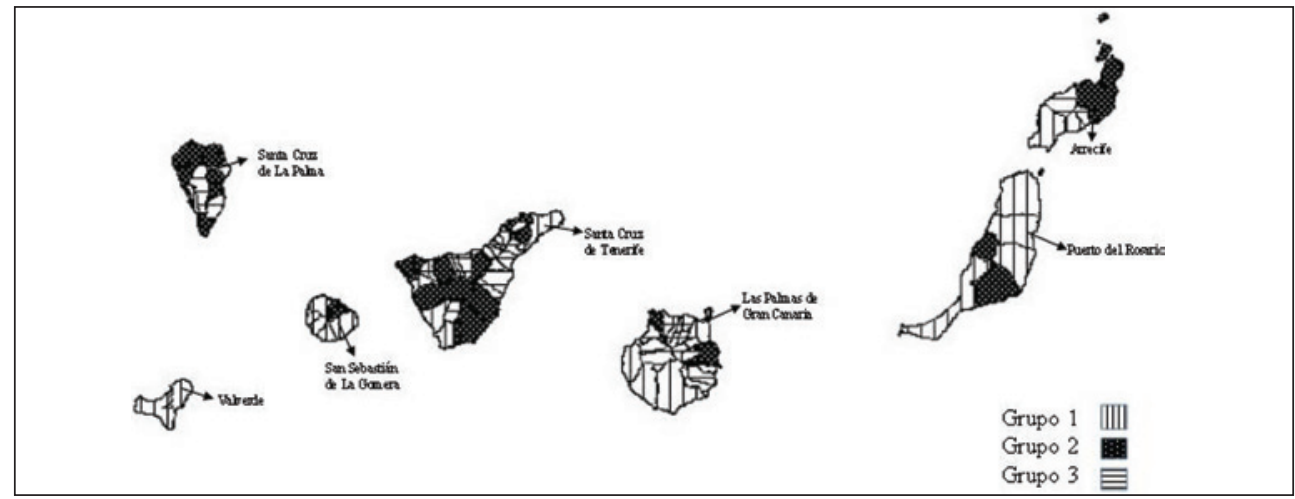

Fuente: INE y elaboración propia. 
Tabla 2

MEDIAS DE LAS VARIABLES INCLUIDAS EN ANÁLISIS CLUSTER POR GRUPO

\begin{tabular}{|l|c|c|c|}
\hline & GRUPO 1 & GRUPO 2 & GRUPO 3 \\
\hline NÚMERO DE MUNICIPIOS & $\mathbf{2 2}$ & $\mathbf{2 8}$ & $\mathbf{3 7}$ \\
\hline VARIABLES EN A. CLUSTER (\%) & \multicolumn{2}{|c|}{ Medias por variable } \\
\hline Movilidad externa & 18,3 & 43,3 & 64,9 \\
\hline ME en los hombres & 19,6 & 45,2 & 66,5 \\
\hline ME en las mujeres & 16,3 & 40,2 & 61,9 \\
\hline ME edad 15-19 & 15,8 & 43,0 & 59,5 \\
\hline ME edad 20-24 & 19,2 & 49,0 & 67,4 \\
\hline ME edad 25-29 & 20,9 & 49,8 & 69,5 \\
\hline ME edad 30-34 & 20,4 & 48,8 & 67,9 \\
\hline ME edad 35-39 & 18,6 & 44,9 & 67,7 \\
\hline ME edad 40-44 & 17,5 & 42,1 & 65,6 \\
\hline ME edad 45-49 & 16,7 & 41,1 & 63,4 \\
\hline ME edad 50-54 & 16,2 & 35,4 & 60,1 \\
\hline ME edad 55-59 & 15,1 & 31,7 & 55,5 \\
\hline ME edad 60 o más & 14,4 & 24,7 & 43,7 \\
\hline ME analfabetos y sin estudios & 15,1 & 31,8 & 53,0 \\
\hline ME primer grado & 16,1 & 39,3 & 59,7 \\
\hline ME segundo grado & 18,3 & 44,1 & 66,7 \\
\hline ME tercer grado & 24,2 & 53,3 & 71,1 \\
\hline ME agricultura, ganadería y pesca & 14,1 & 28,8 & 43,5 \\
\hline ME industria & 90,2 & 86,2 & 88,3 \\
\hline ME construcción & 22,5 & 54,3 & 72,3 \\
\hline ME servicios & 18,1 & 44,5 & 65,4 \\
\hline
\end{tabular}

Fuente: Elaboración propia. Información municipal obtenida del Censo de Población 2001

El resultado más singular de esta clasificación de municipios por modalidad es el hecho de que la movilidad externa en la rama de actividad industrial, independientemente del grupo que se considere, no sólo es la que siempre arroja un nivel porcentual mayor sino que tal porcentaje supera en cualquiera de los casos el $86 \%$ (Tabla 2). La explicación de este suceso se encuentra en que son pocos los municipios canarios que ofrecen al ciudadano la posibilidad laboral en esta rama de actividad por lo que un elevado número de trabajadores en este sector tendrán que desplazarse fuera de su municipio para llevar a cabo su trabajo. Pero debido a que todos los grupos presentan altos porcentajes de movilidad laboral externa en el sector industrial, no parece ésta la razón de la fusión de municipios en uno u otro grupo.

La aplicación del análisis cluster permite conocer los integrantes de cada grupo y con ello las peculiaridades de los municipios que los componen. Así, en la Tabla 3 se proporciona la clasificación de los 87 municipios en los 3 grupos, no sólo en número sino según la isla de pertenencia. Y de estos datos, se puede concluir que mientras la isla más occidental de todas las Canarias y, casualmente, la de menor tamaño -El Hierro- clasifica sus dos únicos muni- 
cipios en el grupo con menor movilidad laboral externa, mientras que las dos islas capitalinas -Tenerife y Gran Canaria- agrupan a la mayoría de sus municipios -17 y 15 municipios, respectivamente- en el grupo 3 o de mayor movilidad externa. Se constata así que, en general, a menor tamaño de la isla, menor población y menor número de municipios por isla, mayor tendencia a que el municipio posea menor movilidad laboral externa; y, por el contrario, a mayor tamaño, más población y mayor división municipal, mayor tendencia a que los municipios de la misma muestren mayor movilidad externa laboral.

\begin{tabular}{|l|c|c|c|}
\hline Isla ( número total de municipios) & GRUPO 1 & GRUPO 2 & GRUPO 3 \\
\hline El Hierro (2 municipios) & 2 & 0 & 0 \\
\hline La Palma (14 municipios) & 1 & 10 & 3 \\
\hline La Gomera (6 municipios) & 4 & 2 & 0 \\
\hline Tenerife (31 municipios) & 5 & 9 & 17 \\
\hline Gran Canaria (21 municipios) & 4 & 2 & 15 \\
\hline Fuerteventura (6 municipios) & 4 & 2 & 0 \\
\hline Lanzarote (7 municipios) & 2 & 3 & 2 \\
\hline
\end{tabular}

Fuente: Elaboración propia. Información municipal obtenida del Censo de Población 2001

El tamaño y la población ocupada de las islas condicionan la diversificación de la actividad económica ofertada en cada una de ellas y, por tanto, los porcentajes de movilidad laboral externa. La mayor diversificación de la actividad económica de las islas mayores provoca mayores posibilidades laborales y, con éstas, mayor ME entre sus municipios; por el contrario, en las islas menores se experimenta una oferta laboral con menos opciones y, por tanto, mayor autocontención laboral que explica los menores niveles de ME. De esta forma, en el análisis de la movilidad externa por islas se observa un patrón de comportamiento similar en Gran Canaria, Tenerife, Lanzarote y la Palma, con más municipios pertenecientes a los grupos 2 y 3 , y por lo tanto mayores tasas de movilidad externa, mientras que La Gomera, Fuerteventura y El Hierro no tienen ningún municipio en el grupo 3, presentando tasas de movilidad externas menores.

Tabla 4

MEDIAS POBLACIONALES POR GRUPO Y VARIABLES SOCIODEMOGRÁFICAS DE LA POBLACIÓN OCUPADA

\begin{tabular}{|c|c|c|c|}
\hline Característica & GRUPO 1 & GRUPO 2 & GRUPO 3 \\
\hline Movilidad laboral externa & 18,3 & 43,3 & 64,9 \\
\hline Población activa & 14945 & 6981 & 4492 \\
\hline Varón & $60 \%$ & $62 \%$ & $63 \%$ \\
\hline Mujer & $40 \%$ & $38 \%$ & $37 \%$ \\
\hline
\end{tabular}


Tabla 4 (continuación)

MEDIAS POBLACIONALES POR GRUPO Y VARIABLES SOCIODEMOGRÁFICAS DE LA POBLACIÓN OCUPADA

\begin{tabular}{|c|c|c|c|}
\hline Característica & GRUPO 1 & GRUPO 2 & GRUPO 3 \\
\hline Edad 15-29 & $29 \%$ & $30 \%$ & $29 \%$ \\
\hline Edad 30-49 & $54 \%$ & $53 \%$ & $56 \%$ \\
\hline Edad 50 o mas & $17 \%$ & $16 \%$ & $16 \%$ \\
\hline Nivel de formación: Analfabeto y sin estudios & $6 \%$ & $9 \%$ & $9 \%$ \\
\hline Nivel de formación: Primer grado & $18 \%$ & $22 \%$ & $22 \%$ \\
\hline Nivel de formación: Segundo grado & $58 \%$ & $55 \%$ & $56 \%$ \\
\hline Nivel de formación: Tercer grado & $19 \%$ & $14 \%$ & $14 \%$ \\
\hline Agricultura, ganadería y pesca & $3 \%$ & $7 \%$ & $7 \%$ \\
\hline Industria & $7 \%$ & $7 \%$ & $8 \%$ \\
\hline Construcción & $11 \%$ & $17 \%$ & $18 \%$ \\
\hline Servicios & $79 \%$ & $69 \%$ & $68 \%$ \\
\hline
\end{tabular}

Fuente: Elaboración propia. Información municipal obtenida del Censo de Población 2001

Por último, con el fin de exponer las características sociodemográficas de cada uno de los grupos obtenidos hemos elaborado la Tabla 4, en donde se observa el reparto entre las modalidades de estas características respecto a la población ocupada media de cada grupo. A modo de ejemplo, el grupo 1, que contiene municipios con menor ME y mayor población ocupada por término medio, está formado por municipios cuyo porcentaje medio de trabajadores varones es del 60\%, mientras que en el grupo 3 estos trabajadores representan el $63 \%$.

El grupo 1 es el formado por los 22 municipios de menor movilidad laboral externa media (menor del 20\%), y mayor población activa media. Conociendo la realidad canaria, se puede deducir que los municipios pertenecientes a este grupo pueden clasificarse, a su vez, en dos subgrupos, por lo que las conclusiones derivadas de la Tabla 4 deben ser asumidas con cierta cautela.

El primer subgrupo lo componen municipios que, o bien son capitales de provincia, o bien municipios con una oferta turística importante. En ambos casos, la oferta de empleo de los mismos es elevada. Concretamente, en este primer subgrupo se encuentran municipios como Santa Cruz de Tenerife y Las Palmas de Gran Canaria, capitales de provincia cuya baja movilidad externa es explicada debido a que sus ocupados trabajan fundamentalmente en su municipio de residencia al tratarse, como se ha indicado, de municipios de alta oferta de empleo. Además, los centros universitarios de la Comunidad Autónoma están ubicados en dichas capitales o muy cerca de ellas, lo que también explica que sea el grupo con mayor porcentaje de trabajadores con formación alta de segundo o tercer grado. También se encuentran en este grupo los municipios del sur de la isla de Tenerife -Adeje, Arona y Santiago del Teide- y de Gran Canaria -Mogán y San Bartolomé-, cuya presencia en este subgrupo se entiende debido a que son municipios de fuerte actividad turística que permite a sus residentes trabajar en el mismo municipio en el que residen. 
El segundo subgrupo estaría compuesto por municipios de islas menores -principalmente de La Gomera y El Hierro- donde las malas comunicaciones, o aquéllas propias de un terreno discontinuo, obligan a la población a trasladar su residencia, en caso de obtener un puesto de trabajo, a aquél en el que se oferta el empleo. Estos municipios presentan baja oferta laboral, que es absorbida por la propia población del municipio, o están definidos por economías muy rurales. En este subgrupo aparecen municipios de islas no capitalinas -como casi todos los de La Gomera y todos los de El Hierro-, cuya escasa movilidad diaria es fruto de ser economías rurales de autocontención laboral (Godenau y Arteaga, 2003) o porque, aún existiendo en alguno de ellos actividad turística, su relevancia es menor y por tanto el número de puestos de trabajo ofertado es también más reducido. A esta segunda modalidad pertenecen, también, casi todos los municipios de Fuerteventura y los del sur de Lanzarote.

El grupo 2 es el formado por los municipios de movilidad externa intermedia. Este tipo de municipios están presentes en todas las islas -capitalinas y no capitalinas-, a excepción de El Hierro, isla más pequeña y más occidental del archipiélago. Los municipios clasificados en este grupo son de actividad económica de cierta relevancia, como son La Laguna, Icod de los Vinos, La Orotava y Guía de Isora en la isla de Tenerife, y Telde y Santa María de Guía en la isla de Gran Canaria. De hecho, en municipios así existe suficiente actividad económica para que sólo un $40 \%$ de sus trabajadores residentes tengan que desplazarse a otro municipio para realizar su actividad laboral diaria.

Por último, el grupo 3 contiene municipios con una alta movilidad externa, municipios que se caracterizan por ser espacios geográficos donde reside una gran parte de la población pero sin una gran oferta laboral. En estos municipios se ha producido un desarrollo urbanístico considerable debido por una parte al incremento de la población y el crecimiento económico en las islas, y por otra al incremento del precio del suelo en las zonas urbanas tradicionales, lo que ha llevado a municipios colindantes a los núcleos urbanos con elevada oferta laboral a convertirse en municipios donde el principal uso del suelo es residencial. De hecho, la mayoría de estos municipios fueron calificados como municipios con una función principalmente residencial en el estudio publicado por el CES de Canarias, que ya hemos citado, y donde se estudiaron los mercados locales de trabajo en Canarias y sus condiciones de movilidad y accesibilidad dentro y entre éstos, haciendo en este caso énfasis en los índices de accesibilidad, el empleo y las estructuras sectoriales de la actividad productiva (Godenau, 2005).

En este grupo se encuentran municipios cercanos a la capital de Tenerife, Santa Cruz de Tenerife, como son los municipios de su vertiente norte, Santa Úrsula, La Victoria de Acentejo, La Matanza de Acentejo, Tacoronte, Tegueste o El Sauzal, y de la vertiente sur como son los de El Rosario, Candelaria y Arafo, donde ciertas zonas de estos municipios presentan las características propias de las llamadas «ciudades dormitorio». También se localizan en este grupo y por razones muy similares -atracción de la actividad económica de la capital de Gran Canaria, Las Palmas de Gran Canaria- los municipios de Santa María de Guía, Arucas, Gáldar, Teror y Santa Brígida entre otros, en los que habitan muchos trabajadores cuyo puesto de trabajo se encuentra en la ciudad capitalina. Existen también municipios de este tercer grupo en La Palma y en Lanzarote, debido a que un mayor desarrollo del sector turístico y una mejora considerable de sus infraestructuras viarias en los últimos años, han permitido generar una mayor movilidad laboral externa, a pesar de ser islas menores y con limitada actividad económica. 


\section{CONCLUSIONES}

La movilidad laboral diaria es un fenómeno complejo, siendo muchas las variables sociodemográficas, aunque no las únicas, que ayudan a comprender este comportamiento de incidencia creciente. De esta forma, este estudio ha pretendido un acercamiento a las características sociodemográficas de la población ocupada en Canarias atendiendo a su movilidad laboral diaria externa e interna, teniendo en cuenta las particularidades de un territorio discontinuo, ya que la Comunidad Autónoma de Canarias es un archipiélago. Además, se hace una clasificación de sus municipios teniendo en cuenta su movilidad laboral externa, con la información extraída del Censo de Población (INE, 2001).

En términos agregados, la movilidad externa en Canarias presenta peculiaridades similares a la movilidad externa en el conjunto del territorio nacional. Como se ha podido comprobar a lo largo del artículo, la movilidad externa e interna entre el lugar de residencia y el de trabajo, difiere en función de las características sociodemográficas de la población de igual forma que en estudios hechos para el territorio nacional y para otras comunidades autónomas. De hecho, analizando la relevancia de la movilidad externa por género, edad, formación y tipo de actividad económica, puede concluirse que el perfil de la movilidad externa en Canarias es: hombre con edad inferior a 50 años, con estudios superiores, y dedicado a actividades económicas de construcción, hostelería, transportes, educación o sanidad.

Las características sociodemográficas y espaciales analizadas influyen en la movilidad laboral en Canarias de forma muy similar a como lo hacen en el resto del territorio nacional. La única diferencia relevante a destacar es la mayor movilidad externa que representa el sector hostelería en las islas, cuestión que cabía esperar debido a que el turismo y, por lo tanto, también la hostelería, es la principal actividad económica en las islas, lo que explica que el trabajador se desplace a los centros turísticos demandantes de mano de obra desde su lugar de residencia, que habitualmente es un municipio cercano.

En el estudio de la movilidad externa por islas se observa un patrón de comportamiento similar en Gran Canaria, Tenerife, Lanzarote y la Palma, con mayores tasas de movilidad externa, que se aproximan mucho al comportamiento de la movilidad externa en los estudios realizados en otros contextos geográficos, mientras que La Gomera, Fuerteventura y El Hierro presentan tasas de movilidad externas menores.

En el análisis abordado en la última sección de este artículo se deja constancia de que las singularidades espaciales del municipio en que se reside ejercen una especial relevancia sobre la movilidad laboral externa de los ocupados. El tipo de oferta laboral de cada municipio y la de los municipios colindantes determinan en gran medida la movilidad laboral diaria de sus trabajadores. En el caso de las Islas Canarias, muchos habitantes de las islas menores se ven obligados a trasladar su residencia a alguna de las islas mayores o capitalinas para ocupar un puesto de trabajo; lo que podría ser un desplazamiento diario en un espacio geográfico continuo, resulta en este caso imposible. Sin embargo, también se podría hacer la lectura contraria: debido a que se trata de un espacio geográfico discontinuo, cada isla «funciona» como un «país independiente» en el que las regiones más activas económicamente ejercen de factor de atracción a los trabajadores de las regiones con menos opciones laborales, por lo que más que hablar de un comportamiento canario único en movilidad laboral se debería tratar de siete comportamientos diferenciados. 


\section{AGRADECIMIENTOS}

Las autoras desean hacer constar su agradecimiento a los revisores anónimos por sus valiosas aportaciones que han contribuido significativamente a mejorar la calidad de este artículo.

\section{BIBLIOGRAFÍA}

ALONSO, M.P., BEAMONTE, M.A., GALLARDO, M.P. y SALVADOR, M. (2007): «La movilidad laboral en Aragón a escala comarcal». XXXIII Reunión de Estudios Regionales, León, 15 y 16 de noviembre de 2007.

ALBERTOS, J.M., NOGUERA, J., PITARCH, M.D., y SALOM, J. (2007): «La movilidad diaria obligada en la Comunidad Valenciana entre 1991 y 2001: cambio territorial y nuevos procesos». Cuadernos de Geografía de la Universidad de Valencia, nº 81-82, 93-118.

ALDENDERFER, M.S. y BLASHFIELD, R. (1984): Cluster Analysis. Series: Quantitative Applications in the Social Sciences. A Sage University Paper, Universidad de Iowa.

BARRIOS, M.C., GODENAU, D. y SCHORN, J. (2009): «Los mercados locales de trabajo y sus condiciones de accesibilidad en Tenerife». Boletín de la Asociación de Geógrafos Españoles, n 49, 67-82.

CASADO DÍAZ, J.M. (2000): Diferencias de género en los desplazamientos cotidianos por razones laborales. Documentos de trabajo: Serie EC (Instituto Valenciano de Investigación Económica), nº 3 .

GARCÍA, J.C. (2008): «Incidencia de las características sociodemográficas en la movilidad metropolitana: El caso de Madrid». Anales de Geografía, vol. 28, nº 1, 53-83.

GODENAU, D. (2005): Elementos comunes y diferenciales de las estructuras económicas y sociodemográficas insulares. Informe Anual 2005. CES de Canarias.

GODENAU, D. y ARTEAGA, S. (2003): La movilidad en los mercados locales de Tenerife. Cabildo Insular de Tenerife. Área de Desarrollo Económico.

GUTIERREZ, J. y GARCÍA, J.C. (2005): «Cambios en la movilidad en el área metropolitana de Madrid: el creciente uso del transporte privado». Anales de Geografía de la Universidad Complutense, vol. 25, 331-351.

MIRALLES-GUASCH, C. y CEBOLLADA, A. (2009): «Movilidad cotidiana y sostenibilidad, una interpretación desde la geografía humana». Boletín de la Asociación de Geógrafos Españoles, $\mathrm{n}^{\circ}$ 50, 193-216.

PAZOS, M. (2005): «Estudio de la movilidad diaria en España: limitaciones en las fuentes y alternativas propuestas». Eria, $\mathrm{n}^{\circ} 66,85-92$.

ROQUER, S. (2007): «Movilidad residencia-trabajo y características sociodemográficas de la población española. Un estudio de sus relaciones a través del Censo de 2001». Boletín de la Asociación de Geógrafos Españoles, n 44, 187-214.

ROQUER, S. y BLAY, J. (2002): «El Censo de población de 2001: análisis y valoración de los principales cambios introducidos». Revista de Geografía, $2^{\mathrm{a}}$ época, no 1, 107-118. 
SALOM, J. y DELIOS, E. (1998): «La movilidad laboral femenina en el País Valenciano: Los factores sociales y laborales en las diferencias de género». Cuadernos de Geografía, $\mathrm{n}^{\circ} 64,485-511$.

SALOM, J. y CASADO, J.M. (2007): «Movilidad cotidiana y mercados locales de trabajo en la comunidad valenciana, 1991-2001». Boletín de la Asociación de Geógrafos Españoles, $\mathrm{n}^{\mathrm{o}} 44,5-28$. 